\title{
El canto intemporal del ruiseñor
}

The timeless song of the nightingale

\section{A canção intemporal do rouxinol}

\section{María Luz Fernández Fernández ${ }^{1}$}
${ }^{1}$ Enfermera. Licencia en Historia. Máster U. en Historia Contemporánea. Profesora de la Facultad de Enfermería de la Universidad de Cantabria.
Correspondencia: Remitirse al correo electrónico
Correo electrónico de contacto: marialuz.fernandez@unican.es

Cómo citar este artículo: Fernández-Fernández, M.L (2021). El canto intemporal del ruiseñor. Cultura de los Cuidados (Edición digital), 25 (No esp.). Recuperado de http://dx.doi.org/10.14198/cuid.2021.esp.06

\section{RESUMEN}

Cuando la Organización Mundial de la Salud declaró el 2020 como año Internacional de las Enfermeras y las Matronas, conmemorando el Bicentenario de Florence Nightingale, nadie pudo imaginar, que la aparición de un virus denominado COVID19, pondría en jaque a la salud mundial situando a la profesión enfermera en primer plano. Es en este escenario donde cobra relevancia la figura de Nightingale, que en este artículo se presenta desde otra óptica con el objetivo de analizar de forma metafórica, tomando como referencia el significado de su apellido en español, ruiseñor, las características que han contribuido a su pervivencia en el tiempo. El resultado final muestra, que hay ciertas afinidades entre el pájaro cantor y Nightingale, como la conexión con la naturaleza, buscando la colaboración con los mejores o la manera en la que se hizo escuchar, con sus escritos, aportando datos. Y lo hizo sin dejarse ver, lo que no disminuyó su protagonismo ya que al igual que el ruiseñor es el protagonista único de la noche, el canto de Nightingale ha trasvasado la línea del tiempo. Este trabajo pone de manifiesto, que Nightingale es una fuente 
inagotable ya que se conjugan en ella numerosas vertientes relacionadas con el sentido de nuestra profesión enfermera, el cuidado holístico, de manera, que es interesante explorar su figura desde otros enfoques.

Palabras clave: Florence Nightingale; Enfermería; Ruiseñor; Vocalización, Animal; Naturaleza.

\section{ABSTRACT}

When the World Health Organization declared 2020 as the International Year of Nurses and Midwives, commemorating the Bicentennial of Florence Nightingale, no one could imagine that the appearance of a virus called COVID19 would put global health in check, placing the profession nurse in the foreground. It is in this scenario that the figure of Nightingale becomes relevant, which in this article is presented from another perspective with the aim of metaphorically analyzing, taking as a reference the meaning of his surname in Spanish, nightingale, the characteristics that have contributed to his survival in the time. The final result shows that there are certain affinities between the songbird and Nightingale, such as the connection with nature, seeking collaboration with the best or the way in which he made himself heard, with his writings, providing data. And he did it without being seen, which did not diminish his prominence since like the nightingale is the only protagonist of the night, Nightingale's song has pierced the timeline. This work shows that Nightingale is an inexhaustible source since it combines numerous aspects related to the meaning of our nursing profession, holistic care, so that it is interesting to explore its figure from other approaches.

Keyworks: Florence Nightingale; Nursing; Nightingale; Animal Vocalization; Nature
Quando a Organização Mundial de Saúde declarou 2020 como o Ano Internacional dos Enfermeiros e Parteiras, comemorando o Bicentenário de Florence Nightingale, ninguém poderia imaginar que o surgimento de um vírus chamado COVID19 colocaria a saúde global em controlo colocando a profissão de enfermeiro na vanguarda. É neste cenário que a figura de Rouxinol se torna relevante, que neste artigo é apresentada de outra perspetiva com o objetivo de analisar metaforicamente, tendo como referência o significado do seu sobrenome em espanhol, rouxinol, ases características que têm contribuído para a sua perviviência ao longo do tempo.

O resultado final mostra que existem certas afinidades entre o pássaro canoro e o Rouxinol, como a ligação com a natureza, procurando a colaboração com o melhor ou a forma como foi ouvido, com os seus escritos, fornecendo dados. E fê-lo sem ser visto, o que não diminuiu a sua proeminência, uma vez que, como o rouxinol é o único protagonista da noite, o canto de Rouxinol transcendeu a linha do tempo. Este trabalho mostra que Nightingale é uma fonte inesgotável, pois combina inúmeros aspetos relacionados com 0 significado da nossa profissão de enfermeiro, cuidados holísticos, por isso é interessante explorar a sua figura a partir de outras abordagens.

Palavras-chave: Florence Nightingale; Enfermagen; Rouxinol; Vocalização Animal; Natureza

\section{INTRODUCCIÓN}

El año 2020 que acaba de concluir, será especialmente recordado por el impacto de la pandemia del COVID 19 a nivel mundial, aunque también, resulta significativo para el colectivo enfermero, que lo inició con la expectativa de visibilizar y poner en valor su aportación profesional en 
el mantenimiento y bienestar de la salud de la población. Esto se enmarca en la decisión que se tomó en el contexto de la $72^{\mathrm{a}}$ Asamblea Mundial de la Salud en 2019 cuando por unanimidad, se declaró el 2020 como el Año Internacional de las Enfermeras y las Matronas. Una cuestión, que defendió el propio Director General, Doctor Tedros Adhanom Ghebreyesus con estas palabras: "La OMS se enorgullece de proponer el año 2020 como el Año de la Enfermera y la Matrona. Estas dos profesiones de la salud son invaluables para la salud de las personas en todas partes. Sin enfermeras y matronas, no lograremos los Objetivos de Desarrollo Sostenible ni la cobertura sanitaria universal”. La elección de este año no fue casual ya que se enmarca, en la celebración del bicentenario del nacimiento de la que se considera como la fundadora de la Enfermería profesional, Florence Nightingale (1820-1910).

Y es esta circunstancia, la que una vez más nos hace retomar la figura Nightingale y la repercusión de su obra, cuyo arranque esencial se sitúa en los resultados estadísticos de su eficaz intervención y la de sus treinta y ocho enfermeras, con los soldados enfermos $y$ heridos en el escenario de la Guerra de Crimea (1853-1856). Un conflicto, en el que el ejército británico junto con el francés y el del reino del Piamonte, participaron como aliados de Turquía en su enfrentamiento contra el Imperio Ruso. A partir de aquí, la popularidad de Nightingale fue en crescendo y desde la oportunidad que le permitió su círculo social y sus bastos conocimientos, realizó oportunas aportaciones a través de numerosos informes y cartas a diferentes políticos, autoridades sanitarias y militares, además de otras personas de relevancia científica e intelectual. Todo ello contribuyó a la realización de numerosas reformas en el ámbito sanitario tanto civil como militar a lo que hay que añadir, la creación de la Escuela de Enfermeras en el Hospital Santo Tomás de Londres en 1860, que marcará un antes y un después para la profesión enfermera.

Esta enfermera y científica, como constaba en el lema del I Congreso Virtual celebrado en Santa Cruz de Tenerife en mayo de 2020 con motivo del Bicentenario de su nacimiento, puso de manifiesto, que lo escrito permanece, ya que deja constancia y que la comunicación de los resultados mediante la aplicación estadística constituye una evidencia esencial para poner de manifiesto la validez y eficacia de las intervenciones enfermeras.

El vasto legado de su obra, sus diarios, la constancia de sus acciones en la prensa escrita de la época y las aportaciones de sus biógrafos, el primero de ellos Sir Edwar Cook, quién en 1913 publica “The life of Florence Nightingale”, y uno de los últimos, el del escritor y crítico británico Mark Bostridge, "Florence Nightingale. The Woman and her Legend", publicada en Londres en 2008, han contribuido a acrecentar su leyenda, aunque también, a acercarnos de manera más objetiva al personaje. Así se aprecia en la obra referenciada de Bostridge reeditada este año 2020 con motivo de los doscientos años del nacimiento de Florence, donde el autor, apoyado en la recopilación de material inédito, entre el que se encuentran documentos familiares, trata de separar la mujer de la leyenda. Esta cuestión se expone en un artículo del Churs Time, Club de Lectura: Florence Nightingale, por Mark Bostridge, en el que Michael Wheeler recomienda la lectura del libro.

Sin lugar a dudas, Florence Nightingale es un personaje que ha generado numerosos artículos e investigaciones y no solo desde el ámbito de la disciplina enfermera, sino también desde la óptica de otras disciplinas como las Matemáticas, la Estadística o la Sociología. Y es en este 
ultimó área, donde se encuadra el trabajo de la Profesora emérita de Sociología de la U. de Guelph (Canadá), Lynn McDonald, editora de la serie "The Collected Works of Florence Nightingale”. Una colección de dieciséis volúmenes en la que se recoge un análisis exhaustivo y detallado de su vida, la influencia de su obra, así como la repercusión de la misma.

Para cualquier autor, abordar la figura de Nightingale supone un reto, a la par que representa una nueva oportunidad de acercarse a ella desde la perspectiva de una nueva dimensión. Por las características de su trabajo y las aportaciones del mismo en las reformas de la salud de su época, resulta sin duda un personaje que transciende más allá de su tiempo encuadrado en el esplendor del Imperio británico, en la Inglaterra del siglo XIX bajo el reinado de Victoria de Kent (1819-1901). Aunque además de lo expuesto en esta argumentación introductoria, parece oportuno hacer referencia al título del artículo, que toma como elementos del mismo el apellido de Nightingale y su traducción al castellano, ruiseñor. Un ave, que viene formando parte de la prosa literaria, la poesía y la música entre otros géneros, por la belleza de su canto y las peculiaridades del mismo durante la noche, escondido, algo que también ocurrió con Nightingale, siempre presente con sus escritos, la mayor parte de ellos desde casa, postrada tras la enfermedad adquirida en Crimea.

En definitiva, se trata de presentar en este artículo a Florence Nightingale desde otro punto de vista, una mujer que sigue siendo un referente, catalogada a veces de visionaria, cuyo trabajo pervive y atraviesa la línea del tiempo como un canto permanente.

\section{DESARROLLO DEL TEMA}

Nightingale: el ruiseñor y la naturaleza
En el preámbulo de este relato se muestra la línea que conduce el mismo a la par, que se indica porqué, se toma al ruiseñor como referencia en paralelismo al significado del apellido de Florence. Es por ello, que nos acercamos ahora a esta ave cantora para comprender a través de algunas de sus particularidades, esa pervivencia de Nightingale en nuestros días.

El nombre científico del ruiseñor común Luscinia megarhynchos, fue acuñado por primera vez por el pastor alemán y ornitólogo Christian Ludwig Brehm (18871864) y según la Sociedad Española de Ornitología (SEO/BirdLife), se trata de un "pájaro de plumaje discreto y que rara vez abandona la espesura, pese a lo cual resulta fácil de detectar por su inconfundible canto, que emite incansablemente, incluso durante la noche. Por su potencia, variedad y constancia, el canto del ruiseñor es el más sobresaliente de entre todas las aves cantoras(...)”.

Para el Diccionario de la Universidad de Oxford el término Nightingale, ruiseñor, es "un pequeño tordo migratorio con plumaje pardusco, que destaca por su rico y melodioso canto que a menudo se puede escuchar por la noche", añadiendo, que esta palabra procede del inglés antiguo Nightegala, de origen germánico y relacionado con el alemán Nachtigall, desde la base de la noche que significa cantar.

Indudablemente, no se trata de buscar una relación directa de la voz de Nightingale con la que proyecta el canto del ruiseñor como si se tratara de un acto mimético, sino de una metáfora, que sitúa a nuestra referente enfermera como la única que ha sido capaz de traspasar la línea de su tiempo. La cuestión se centra en su proyección a través de sus escritos y no en sus palabras habladas de las que apenas tenemos un registro, se trata de la grabación 
que se hizo en su casa el 30 de julio de 1890 en un cilindro de parafina de Edison. El fin de la misma fue conseguir dinero para el Fondo de Ayuda de los veteranos de la Brigada Ligera y Florence expresa: Cuando ya no sea ni siquiera un recuerdo, solo un nombre, espero que mi voz pueda perpetuar la gran obra de mi vida. Dios bendiga a mis queridos y viejos camaradas de Balaclava y los lleve a salvo a la orilla. Florence Nightingale.

Por tanto, es el amplio lenguaje escrito lleno de indicadores y datos que aportó Nightingale, lo que sitúa su trascendencia y la pervivencia en nuestros días. Sin entrar en las diferencias que constituye el planteamiento de los géneros literarios parece interesante reseñar el artículo de Pablo Castellanos, La emoción ante las palabras: Teoría de Borges sobre el Lenguaje poético. Aquí, el autor, referencia a Borges, quién expone: "El ruiseñor, en todas las lenguas del orbe, goza de nombres melodiosos

(nightingale, nachtigall, usignolo), como si los hombres instintivamente hubieran querido que estos no desmerecieran del canto que los maravilló". El poeta argentino nombra al ruiseñor planteando, que indistintamente del idioma de cada país, se mantiene la belleza y melodía de su canto. Esto mismo ocurre en la profesión enfermera con respecto a la figura de Florence Nightingale, representación e icono de la misma a nivel internacional más allá de las barreras idiomáticas, geográficas $\mathrm{y}$ culturales.

Indudablemente, al igual que Borges, son muchos los poetas que han tomado como referencia al ruiseñor, aunque aquí hablamos de la Philomena, de la poeta andaluza Pilar Paz, cuyo título parte de un pasaje del Cántico espiritual de San Juan de la Cruz: "el dulce ruiseñor forma parte de las delicias de la unión del alma con
Dios(...)puestas en boca de la Esposa, que insta al Amado a gozar del amor en el seno de la naturaleza”. Así lo especifica A. S. Pérez- Bustamante en "La Lengua de los pájaros”. Filomena como símbolo en la poesía de Pilar Paz Pasamar, quién dice sobre su poemario: "Philomena es la palabra como oración, la escritura poética como oración, como medio que tiene el poeta para fundirse con todo lo creado. Pero junto a la espiritualidad también tiene su parte de experiencia humana y directa(...)”. Es en el contexto expuesto en el que alcanza significado F. Nightingale, desde la dimensión espiritual de ese momento en el que expresa en su diario que se sintió llamada por dios, un aspecto que analiza detalladamente la Prof.. Lynn McDonald en “Florence Nightingale: Fe y trabajo”. En el mismo, concretamente en un apartado dedicado a la oración especifica que Nightingale amaba a los pájaros y transcribe sus palabras: No hay nada que haga vibrar mi corazón como la voz de los pájaros, mucho más que la voz humana. Son ángeles llamándonos con sus canciones.

Nightingale puso de manifiesto la trascendencia y el efecto beneficioso de la naturaleza en la salud de las personas y es desde esta óptica, en la que vuelve a cobrar sentido el ruiseñor en su conjunción con el ámbito natural. En esta línea, Carlos Hita, técnico de sonido y autor del audioblog "El sonido de naturaleza” en la edición digital del diario El Mundo, en una publicación, bajo el título "Puesta en escena nocturna", nos traslada el sonido del canto del ruiseñor que aprovecha la noche para convertirse en palabras de Hita, en el protagonista absoluto.

A la par especifica que, aunque no es el mejor cantor entre todas las aves silvestres, "Lo que no se puede negar al ruiseñor es lo que podríamos llamar un gran sentido de la puesta en escena, ya que sabe aprovechar los mejores momentos para 
emitir su inacabable canción, cuando las acústicas son más favorables”. Sin duda, estas palabras pueden aplicarse a Nightingale quién supo utilizar hábilmente su posición, relaciones y recursos para hacerse oír y que sus indicaciones se tuvieran en cuenta.

Ahora que vivimos en un mundo amenazado por el cambio climático y su impacto real en la salud humana, el planteamiento de Nightingale y su teoría del entorno cobra una especial relevancia como puede apreciarse en numerosos artículos de investigación. Sin embargo, antes de detallar este aspecto y su relación con la práctica enfermera, es interesante plasmar el trabajo publicado en la Revista The Auk. Ornithological Advances, "Cambio climático y acortamiento inadaptado de un ala en un ave migratoria de larga distancia”.

Los autores de esta investigación, cuyos datos se han centrado en veinte años de seguimiento de los ruiseñores comunes en el centro de España, donde se han producido cambios medioambientales, muestran que estas "aves han reducido la longitud de sus alas relativa al tamaño corporal a lo largo del período 1995-2014” y que según los resultados obtenidos, "las poblaciones de aves pueden variar desviándose de su óptimo fenotípico, lo que nos ayuda a comprender mejor las restricciones ecológicas que pueden condicionar su adaptación al rápido cambio global”.

Al igual que les ocurre a los ruiseñores, las circunstancias medioambientales tienen una clara repercusión en la salud humana y ya centrados en nuestra actividad enfermera, es oportuno destacar la publicación de la Revista Journal Of Clinical Research \& Bioetics bajo el título "Clinical Application of Nightingale's Theory”. En la misma, los autores de este trabajo exponen la importancia de los principios enunciados por
Florence en su teoría del medio ambiente y su aplicación en la práctica actual resaltando, que los problemas a los que se enfrentan los pacientes están más relacionados con su entorno que con su enfermedad.

Los principios establecidos por Nightingale y cuya aplicación mostró su efectividad real en la atención a los soldados de Crimea, quedaron plasmados en su ya conocida obra "Notas sobre Enfermería. Qué es y qué no es”. En ella, deja constancia de la trascendencia de la adecuada utilización de la luz natural, la ventilación, la limpieza y la visión de la naturaleza entre otros, como elementos imprescindibles en las casas y hospitales para mejorar la situación de los enfermos y de la población. Un aspecto que refleja Jan Findlay Presidenta del Landscape Institute, y Directora del Fira (Landsacape architecture and urban design) con sede en Londres y Birmingham y que representa a un "grupo de arquitectos paisajistas, diseñadores urbanos.... con un espíritu centrado en las personas”. En un artículo publicado en Fira, "El paisaje curativo: la influencia de Florence Nightingale en el diseño de los hospitales”, Findlay, su autora, describe la importancia de la aportación de Nightingale en los cambios de la estructura hospitalaria de la época centrados en una arquitectura saludable en la que cobran importancia los jardines interiores.

\section{Nightingale: un reclamo constante}

$\mathrm{Al}$ referirse al paisaje sonoro de la naturaleza, Carlos Hita lo describe como una gran orquesta donde los distintos instrumentos suenan armónicamente, el viento, la lluvia, el agua, las distintas especies animales como las aves donde se encuentra el ruiseñor, que según Hita es el "protagonista absoluto del espacio sonoro". Ahora bien, si su canto le sitúa en el primer plano, lo que resulta más difícil es divisarlo, ya que si algo le caracteriza es "la simpleza 
de su plumaje y la discreción de su vida, siempre oculta en las espesuras del campo". Nuestro ruiseñor, F. Nightingale, guarda algunas similitudes con esta ave, ya que se ha convertido en la protagonista absoluta de la disciplina Enfermera gracias a la búsqueda e interpretación constante de los datos. Con ellos, puso de manifiesto su personalidad y conocimientos matemáticos haciéndose escuchar, en el contexto de un mundo victoriano marcado por las deplorables condiciones de vida de la población inglesa. Un trabajo, que tras Crimea y como consecuencia de haber adquirido una brucelosis crónica que minó su salud, realizó desde la reclusión de su casa. Sin embargo, esto no constituyó un motivo para emitir continuos informes sobre las condiciones de los hospitales, las casas de pobres y otras cuestiones que ponían en evidencia la necesidad de hacer reformas. En muchas ocasiones se cuestionaba si de verdad estaba tan enferma, ya que quienes la visitaban destacaban su vitalidad lo que según Mark Bostridge, la convirtió en "una de las inválidas más famosas de la historia”.

Puede decirse, que esta inglesa proyectó su saber desde el backestage, de manera, que lo más complejo era verla, pues al igual que el ruiseñor, permanecía oculta, aunque como el bello canto del pájaro, esto no disminuyó la relevancia y el impacto de su trabajo. Sin embargo, todos somos conocedores de la importancia que tienen las imágenes y es en relación a esto, por lo que tratamos aquí el asunto de las fotografías de Nightingale. En lo que a estas concierne, parece oportuno reflejar el artículo del historiador fotográfico Denis Pellerin y su asistente Rebecca Sharpe, publicado en la Revista The classic bajo el título: Florence Nightingale: Los misterios detrás de sus icónicas fotografías.

Los autores llevaron a cabo una investigación dirigida a identificar las fotos en las que aparece, las circunstancias en las que se tomaron e incluso, los fotógrafos que las realizaron, manifestando, que ella no quería ser fotógrafa y trataba de evitarlo.

En cuanto lo referenciado anteriormente, los resultados de este trabajo ponen de manifiesto, que si bien, Florence siempre dijo que tan solo posó una vez a petición de la Reina Victoria, no fue así y aunque su imagen aparece en numerosos lugares, este trabajo pone de manifiesto que fueron dos fotógrafos los que consiguieron ponerla ante su cámara: Claudius Erskine Goodman (1822-1873) y William Edward Kilburn (1818-1891). El primero es el autor de dos fotografías la primera, una de las más icónicas, en la que Nightingale aparece frente a una cortina y la segunda, parece que después del regreso de Crimea, de perfil sin su gorro, se ve su pelo corto y liso. Esto puede confirmarse según Pellerin y Sharpe, porque la Reina Victoria lo menciona en su Diario del lunes 22 de septiembre de 1856: "Llegó la señorita Nightingale, vestida de negro, con un simple gorro, atado bajo la barbilla, el pelo cortado...”. También se aprecia en esta foto su aspecto demacrado e igualmente esto, según los autores, aparece en el libro real: "Es alta y delgada, con hermosos ojos oscuros debe haber sido muy bonita pero ahora se ve muy delgada y desgastada por los cuidados”.

Como se expresado, William Edward Kilburn fue el otro fotógrafo que tuvo la fortuna de inmortalizar a Nightingale en dos ocasiones, en una de ellas aparece “(...) sentada mirando hacia abajo...y en otra de pie junto a un pedestal ornamentado mirando a la derecha(...)”, ambas son muy conocidas y aunque parece que se tomaron entre 1861 y 1863, no se ha podido autentificar la fecha.

Puede decirse que, si bien Nightingale solo se fotografíó en escasas 
Cultura de los Cuidados

ocasiones, son innumerables las reproducciones de su imagen en distintos soportes: tarjetas, sellos, litografías, o papel moneda entre muchos otros, de manera, que la visualización de su figura no ha hecho más que contribuir a su pervivencia manteniéndola como un icono. Sin duda, es evidente que la importancia de su figura ha sido motivo de la obra de escultores y también de algunos artistas, como los que la han plasmado en las vidrieras, la mayoría de ellas ubicadas en lugares de culto religioso y generalmente conocidas. Se hace así referencia únicamente a la que estaba previsto inaugurar el 12 de mayo del 2020, con motivo del bicentenario del nacimiento de Florence en Romsey Abbey, Hampshire, cerca de la que fue su casa en Embley Park, y que debido a la pandemia se ha pospuesto hasta este año. La autora, Sophie Hacker, ha centrado su obra en el momento en el que Nightingale, a punto de cumplir los dieciséis años, sentada en un banco de piedra de su jardín, según escribió en su diario, sintió la llamada de Dios a su servicio. Un momento que la artista ha querido destacar, que se unen muchos de los elementos, que de alguna manera guardan relación con este momento clave en su vida.

Por otro lado, otra vertiente que une a Nightingale con el ruiseñor es que este último destaca por la belleza de su canto nocturno, momento en el que se impone a todos los demás. Y es sobre todo la noche, cuando visitaba a los soldados con una lámpara, el momento en el trasciende su figura y que le valió la denominación de "La Dama de la Lámpara”. El primero que publica una imagen de Florence con la lámpara el 8 de febrero de 1855, fue el The Times, destacando: "Cuando todos los oficiales médicos se han retirado ya y el silencio y la oscuridad descienden sobre tantos postrados dolientes, puede observársela sola, con una pequeña lámpara en su mano, efectuando sus solitarias rondas". Aunque lo que hizo transcender este aspecto fue el poema del estadounidense Henry Wadsworth Longfellow (1807-1882), en Santa Filomena, publicado en The Atlantic Monthly en 1857. La lámpara llega hasta nuestros días como un símbolo que sigue iluminando la profesión enfermera en todo el mundo.

Y bien, se ha tratado de reflejar en este apartado la similitud de algunas de las características entre el apellido de Florence y el ruiseñor destacando, que estas han favorecido su continuidad y contribuyendo a la configuración de su figura como la representación de la profesión enfermera en todo el orbe.

\section{La sinergia de las mentes valiosas}

El músico, científico y naturalista Bernie Krause, nacido en Detroit, Michigan en 1938, publicó en el 2012 el libro: La gran orquesta animal, con el objetivo de mostrar, que hemos aprendido a bailar y cantar gracias a los animales, en lo que describe como "paisajes sonoros", acuñando dos términos, las biofonías y las geofonías, que según este ecologista "han tenido una influencia decisiva en nuestra cultura”. Para Krause, las biofonías, son la organización de las voces colectivas que configuran el hábitat natural poblado por las distintas especies animales, mientras que las geafonías, hacen referencia a los sonidos de las fuerzas de la naturaleza.

En una conferencia TEDglobal en 2013, bajo el título, la voz del mundo natural disertó sobre el hecho de "como cada paisaje sonoro que brota de un hábitat silvestre produce una característica única, que proporciona gran cantidad de información desde muchas perspectivas, desde datos cuantificables, hasta inspiración cultural”. Una vez más, se conjugan los elementos de la naturaleza, que Nightingale tanto defendió, y la trascendencia de los datos 
como argumentación efectiva de que la implementación de medidas adecuadas permite obtener resultados satisfactorios. En este sentido, Florence escribió: “Lograr a través de los ojos lo que no somos capaces de trasmitir a las mentes de los ciudadanos a través de sus oídos insensibles a las palabras”. Sin embargo, fue consciente, para lograrlo, de que era imprescindible la colaboración y el trabajo de personas expertas en distintos campos como la Estadística, Matemáticas, Medicina o la Sociología. Y así, como si se tratara de una gran orquesta, donde los distintos instrumentos se conjugan de manera armónica para como en este caso, mejorar las condiciones de salud, Nightingale trabajó con eminentes personalidades del campo científico de su época. Así, entre ellas destacar, al estadístico William Farr (18071873); la matemática Ada Lovelace (18151872); Harriet Martineau (1802-1876), considerada la primera socióloga y que trabajó con Nightingale en el libro, “Inglaterra y sus soldados" en 1859 o Elizabeth Blackwell (1821-1910), primera graduada en Medicina y pionera en la educación médica de la mujer.

Es difícil saber si fue su mente observadora, aunque lo que puede comprobarse, es su capacidad para conjugar los talentos de las mentes valiosas de su tiempo. Este aspecto puede verse reflejado en el artículo: Florence Nightingale's networks Women. Power, and scientific collaboration, donde Lorenzo Arribas y Cacheiro, mediante la construcción de un egonet, que definen como "una red social centrada en un individuo o entidad, que se conoce como ego ...”, simbolizan las relaciones de Nightingale a partir del análisis de su producción a lo largo de toda su vida. Los resultados de su trabajo se muestran en un mapa interactivo en el que se aprecia según las autoras, "una amplia gama de relaciones de colaboración de Nightingale y sus conexiones influyentes", hombres y mujeres del ámbito político; reformadores sociales; estadísticos, científicos y académicos, además de la familia y amigos influyentes. En la conclusión especifican, que Nightingale se relacionó con hombres poderosos como el ya referenciado William Farr, y el Dr. en Medicina Jhon Sutherland, con quienes trabajó en "Notas sobre asuntos que afectan la salud, la eficiencia $\mathrm{y}$ administración hospitalaria del ejército británico”. Pero también destacan su relación con mujeres poderosas, como las mencionadas anteriormente, que la "ayudaron a hacer posible su trabajo innovador".

Una de las personas, cuya investigación ha contribuido a esclarecer todos los aspectos del trabajo de Nightingale ha sido la Prof. Lynn McDonald, a quién ya se ha hecho mención en este texto. Ahora, se trata de aportar algunas de las cuestiones que refleja en el artículo. "The real godos and the oversell”. Este, publicado en la Revista Significance, de la Royal Statistical Society, en marzo de 2020, forma parte de la edición especial para conmemorar el bicentenario del nacimiento de Nightingale. En su disertación McDonald concluye exponiendo, que Florence “...orientó su trabajo a salvar vidas, especialmente de soldados, pobres enfermos, víctimas del hambre, pueblos indígenas, madres $\mathrm{y}$ enfermeras, personas, que no eran en general, prioridades en las políticas públicas del momento...”. Igualmente, especifica que no trabajó sola, ya que contó: “con un equipo de expertos comprometidos para abordar estos temas(...)”.

McDonald expresa también, la preocupación de Nightingale no solo por la recopilación de los datos, sino porque estos fueran “accesibles y persuasivos” y aunque según esta autora, no todo fueron resultados positivos, la define como una mujer 
“adelantada a su tiempo", que consiguió grandes logros.

Para cerrar este apartado, referirnos a RJ Anderws, artista y narrador de datos, autor de la obra: "Info We Trust: How to Inspire the World with Data”, publicada en 2019. Así mismo, es el autor de la portada del número de la Revista Significance, ya citada anteriormente, creando una ilustración inspirada en según sus palabras en esta "estadística, enfermera y reformadora”.

En la Data Visualization Society, dedica un apartado a Nightingale, en el que Andersws, escribe "Florence Nightingale is a Design Hero", en el que analiza detalladamente su trabajo con los datos y destaca, que "(..) manejó un concierto de disciplinas de diseño, al menos: el diseño estratégico, comunicación, organización, servicio, arquitectura, información, finanzas y gráficos(...)", destacando que no fue esto lo que la convirtió en heroína sino el noble objetivo que perseguía, reformar los hospitales y como también expresó McDonald, mejorar las condiciones de salud no solo militares sino también de la población.

\section{CONCLUSIÓN}

A lo largo de todo este discurso se pone de manifiesto, que existen múltiples y variadas razones argumentativas para comprender la dimensión de Florence Nightingale, un personaje, que parece siempre una fuente inagotable debido a las múltiples facetas que se conjugan en su vida y obra. En este caso, se ha tratado de aportar una nueva visión a partir del significado de su nombre en español, ruiseñor y se ha podido comprobar, desde un punto metafórico, que ciertamente, existen algunas conexiones.

El análisis de las características del ruiseñor, desde el contexto de su ámbito natural y las peculiaridades de su canto, han servido de referencia para mostrar algunas de los rasgos de la personalidad de Nightingale y la repercusión de su trabajo, como elementos que han contribuido a su imagen icónica y la pervivencia más allá de su tiempo. Desde su llamada divina a la importancia de la naturaleza para mantener la salud, sus continuos escritos y la preocupación constante por mostrar a través de los datos, la evidencia de sus intervenciones, constituyen los elementos claves de su éxito. Aunque sin olvidar, que en ello tuvo una gran relevancia la magnífica conexión de relaciones y trabajo con las personas más eminentes de la época en los distintos campos científicos y de poder.

Sin duda, su legado sigue siendo fuente de aprendizaje e inspiración y ha quedado claro en el contexto de la pandemia actual, entre otras cuestiones, por su insistencia en el lavado de manos, la limpieza o la ventilación. Además, con motivo su bicentenario, en el marco del Año de las Enfermeras y las Matronas declarado por la OMS este pasado 2020, Nightingale ha sido objeto de numerosos artículos periodísticos, uno de ellos, del periódico The Guardian en mayo de 2020, donde Carola Hoyos analizaba cómo habría abordado Florence Nightingale el Covid19.

Entre las distintas cuestiones Hoyos refiere que hoy "no estaría caminando con una lámpara entre los pacientes de los hospitales.... Sino mirando fijamente su computadora portátil, su teléfono inteligente con miles de mensajes de texto con las personas influyentes del momento, la Reina, el Primer ministro, hasta matemáticos y epidemiólogos. Su computadora estaría llena de hojas de cálculo y un animado debate en Twitter sobre la confianza de los datos”.

A pesar de la distancia en el tiempo, las diferencias entre el mundo decimonónico y la actualidad, se aprecia, que muchos de los 
Revista científica de la Asociación de Historia y Antropología de los Cuidados (Universidad de Alicante)

planteamientos de Nightingale tienen total vigencia y que sus estrategias, pueden resultar igualmente válidas hoy.

La actual pandemia ha llevado a los profesionales enfermeros a vivir situaciones de cansancio y agotamiento, como consecuencia de los oídos sordos de los responsables sanitarios entre otros, ante nuestra insistencia en concreto por la escasez de enfermeros para dar respuesta a la necesidad de cuidados de la población. Y si esto era así hace años, cuando sucede una situación como la que estamos viviendo, es “la gota que colma el vaso”. En esta línea es interesante hacer referencia a las palabras de la Prof. de Política de Enfermería y ex Decana de la Facultad de Enfermería y Partería de Florence Nightingale del King's College Londres, Anne Marie Rafferty, quién en una entrevista de The Guardian en octubre de 2020, expuso, que el "Covid debería ser un corto circuito para los males que aquejan a la enfermería”.

Por otro lado, este relato pone de manifiesto, que aún quedan muchas áreas por investigar relacionadas con Nightingale, quizás porque los estudios sobre la figura más representativa de la Enfermería se han centrado solo en algunas cuestiones de la profesión. Aunque si de verdad se tuviera en cuenta, que el cuidado enfermero tiene sentido desde una visión holística esto nos permitirá apreciar la riqueza de sus aportaciones.

Así, y para no perder el hilo conductor de este texto ligado a Nightingale y el ruiseñor, parece oportuno, transcribir la referencia de la cuentacuentos y escritora Estrella Ortíz Arroyo. Esta, en una de sus publicaciones, transcribe una Conferencia sobre "El ruiseñor de Machado" y hace referencia a un cuento de Gotthold Lessing, en el que "nos invita, por su propio bien y el de los demás, a seguir cantando y no apagar nunca la voz":

- Vamos, querido ruiseñor, canta, le pidió al pájaro callado, una bella noche de primavera.

- $\quad$ iAyi - exclamó el ruiseñor- Las ranas hacen tanto ruido que me han quitado las ganas de cantar. ¿No las oyes?

- Claro que las oigo- repuso el pastor-justamente las oigo debido a tu silencio.

Nightingale, sigue resonando en nuestros oídos a través de su legado escrito, como un canto intemporal, que nos anima a las enfermeras a no apagar nuestra voz y para ello, nada como sus palabras, que una vez más, se adelantan a su tiempo:

Estoy convencida de que los héroes más grandes son aquellos que cumplen con sus deberes diarios y asuntos domésticos mientras el mundo va girando como una peonza enloquecida.

\section{BIBLIOGRAFÍA}

AliSher, A. N., Atta, S., Yasin, I., \& Sohail, M. A. (2019). Clinical Application of Nightingale's Theory. International Journal of Nursing Care, 7(1), 13-16. Recuperado de https://www.longdom.org/openaccess/clinical-application-of-nightingalestheory-2155-9627-1000329.pdf

Andrews, R.J. (2019). Florence Nightingale is a Design Hero: May her light forever shine bright. Recuperado de https://medium.com/nightingale/florencenightingale-is-a-design-hero-8bf6e5f2147 Andrews, R. J. (2020). Let there be light: A tribute to Florence Nightingale. Significance, 17(2), 16-17. https://doi.org/10.1111/1740-9713.01373 BBC News. (2021). Florence Nightingale window installed in Romsey Abbey. Recuperado

de 
https://www.bbc.com/news/uk-englandhampshire-54466201

Castellanos, P. (2012). La emoción ante las palabras: teoría de Borges sobre el lenguaje poético. Literatura: teoría, historia, crítica, 14(2), 313-65. Recuperado de https://revistas.unal.edu.co/index.php/lthc/a rticle/view/37132/39160

ConSalud,es (mayo, 2019). Tedros Adhanom: Año Internacional de la Enfermera. Recuperado de https://www.consalud.es/barometrosanitario/positivo/tedros-adhanom-anointernacional-de-laenfermera_64282_102.html

Costa, R., Padilha, M. I., Amante, L. N., Costa, E., \& Bock, L. F. (2009). O legado de Florence Nightingale: uma viagem no tempo. Texto \& ContextoEnfermagem, 18(4), 661-669.

CHURCH TIMES. (2020). Book club: Florence Nightingale, by Mark Bostridge. Recuperado de https://www.churchtimes.co.uk/articles/202 0/7-august/books-arts/reading-groups/bookclub-florence-nightingale-by-markbostridge

Cué, E. (2017). Bernie Krause: «Tenemos que aprender a cerrar el pico y proteger activamente nuestro entorno». $\mathrm{ABC}$ Cultura. Recuperado de https://www.abc.es/cultura/arte/abci-berniekrause-tenemos-aprender-cerrar-pico-yproteger-activamente-nuestro-entorno201705280056_noticia.html De Hita, C. (2008). El sonido de la Naturaleza: Puesta en escena nocturna.

El Mundo.es. Recuperado de https://www.elmundo.es/especiales/2008/05 /ciencia/sonido_naturaleza/sonidos_17_05 2013.html

De Hita, C. (2009). El sonido de la Naturaleza: El canto del ruiseñor. El mundo.es. Recuperado de https://www.elmundo.es/especiales/2008/05 /ciencia/sonido_naturaleza/sonidos_03_04 2009.html
De la Salud, A. M. (2019). Proyecto de presupuesto por programas 2020-2021: informe del Comité de Programa, Presupuesto y Administración del Consejo Ejecutivo a la 72. ${ }^{a}$ Asamblea Mundial de la Salud (No. A72/63). Organización Mundial de la Salud. Recuperado de https://www.who.int/es/about/governance/ world-health-assembly/seventy-secondworld-health-assembly

De la Salud, O. (2020). 2020 año internacional enfermeras y matronas OMS. Recuperado de https://www.who.int/es/newsroom/campaigns/year-of-the-nurse-and-themidwife-2020

Evens, J. (2021). The Calling Window by Sophie Hacker: Interview with Sophie Hacker. ArtWay. Recuperado de https://www.artway.eu/artway.php?id=1105 \&lang=en\&action=show \&type=current

FIRA (2020). The Healing Landscape: The Influence of Florence Nightingale on Hospital Desing. Recuperado de https://www.fira-la.com/the-healinglandscape-the-influence-of-florencenightingale-on-hospital-design

Hammer, J. (2020). The defiance of Florence Nightingale. Smithsonian Magazine. Recuperado de https://www.smithsonianmag.com/history/t he-worlds-most-famous-nurse-florencenightingale-180974155/

Hoyos, C. (2020). How would Florence Nightingale have tackled Covid-19? The Guardian. Recuperado de https://www.theguardian.com/society/2020/ may/05/how-would-florence-nightingaletackled-covid-19-200-year-anniversary

Lorenzo-Arribas, A., \& Cacheiro, P. (2020). Florence Nightingale's network: Women, power, and scientific collaboration. Significance, 17(2), 22-25. McDonald, L. (2020). The real goods and the oversell. Significance, 17(2), 18-21. 
McEnroe, N. (2020). Celebrating Florence

Nightingale's bicentenary. Lancet (London, England), 395(10235),1475.

Monacchi, D., \& Krause, B. (2017). Ecoacoustics and its expression through the voice of the arts: An essay. Eco Acoustics: The Ecological Role of Sounds, 297-311.

Mourier, A. S. P. B. (2011). La lengua de los pájaros: Filomena como símbolo en la poesía de Pilar Paz Pasamar. Salina: revista de lletres, (25), 159-186.

Mulholland, H. (2020). Anne Marie Rafferty: 'Covid should be circuit breaker for the ills plaguing nursing'. The Gurdian. Recuperado de https://www.theguardian.com/society/2020/ oct/21/anne-marie-rafferty-covid-circuitbreaker-ills-plaguing-nursing-rcn Nightingale, F., \& McDonald, L. (2009). Florence Nightingale: Collected Works of Florence Nightingale (Vol. 13). Wilfrid Laurier Univ. Press.

Ortiz, E. (2020). El ruiseñor de Machado. Recuperado de https://estrellaortiz.es/publicaciones/articulo s/el-ruisenor-de-machado/

Pellerin, D. (2020). Florence Nightingale: The Mysteries Behind Her Iconic Photographs. The Classic. Recuperado de https://theclassicphotomag.com/florencenightingale-the-mysteries-behind-hericonic-photographs/

Remacha, C., Rodríguez, C., de la Puente, J., \& Pérez-Tris, J. (2020). Climate change and maladaptive wing shortening in a longdistance migratory bird. The Auk, 137(3), ukaa012. Recuperado d:

https://doi.org/10.1093/auk/ukaa012

https://academic.oup.com/auk/articleabstract/137/3/ukaa012/5814682?redirected From=fulltext

University of Nottingham. (2020). Manuscripts and Special Collections. Florence Nightingale Comes Home. Recuperado de https://www.nottingham.ac.uk/manuscriptsa ndspecialcollections/exhibitions/online/flor ence-nightingale/index.aspx

Velázquez Aznar, A., \& Dandicourt Thomas, C. (2010). Florence Nightingale. La dama de la lámpara (1820-1910). Revista cubana de enfermería, 26(4), 166-169. Recuperado de https://ciberindex.com/index.php/ie/article/ view/e21503/e21503

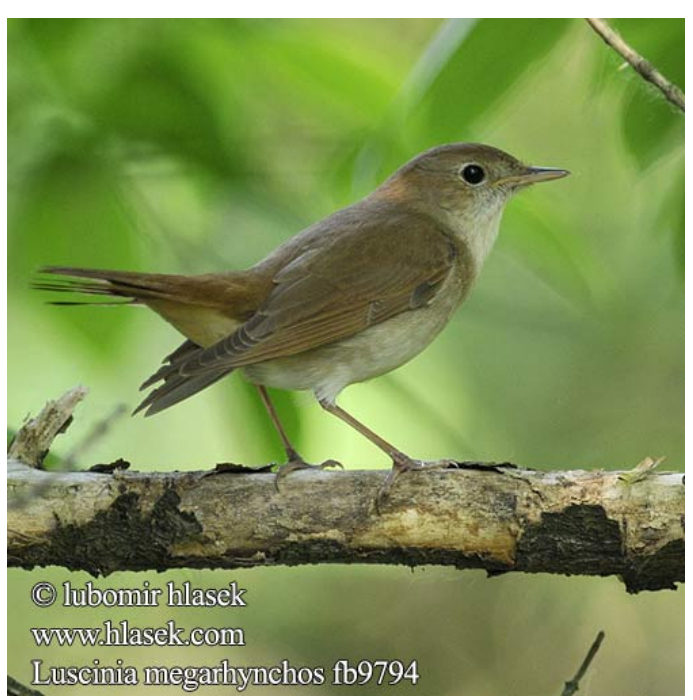

